\title{
Prognostic Significance of the Tumor-Stroma Ratio in Epithelial Ovarian Cancer
}

\author{
Ying Chen, ${ }^{1,2,3}$ Lei Zhang, ${ }^{1,2,3}$ Wenxin Liu, ${ }^{1}$ and Xiangyu Liu ${ }^{1}$ \\ ${ }^{1}$ Department of Gynecologic Oncology, Tianjin Medical University Cancer Institute and Hospital, Tianjin 300060, China \\ ${ }^{2}$ Key Laboratory of Cancer Prevention and Therapy, Tianjin 300060, China \\ ${ }^{3}$ National Clinical Research Centre of Cancer, Tianjin 300060, China \\ Correspondence should be addressed to Ying Chen; lychenying2004@126.com
}

Received 6 June 2015; Revised 31 August 2015; Accepted 31 August 2015

Academic Editor: Genichiro Ishii

Copyright (C) 2015 Ying Chen et al. This is an open access article distributed under the Creative Commons Attribution License, which permits unrestricted use, distribution, and reproduction in any medium, provided the original work is properly cited.

\begin{abstract}
Tumor-stroma ratio (TSR) has recently been identified as a promising prognostic parameter for several solid tumors. This study aimed to evaluate the prognostic role of TSR in epithelial ovarian cancer (EOC) and 838 EOC patients were enrolled in this study. TSR was estimated on hematoxylin-and-eosin-stained tissue sections from the most invasive part of the primary tumor. Patients were classified as stroma-rich or stroma-poor according to the proportion of stroma $\geq 50 \%$ or $<50 \%$. Chi-square test analysis revealed that TSR were significantly associated with FIGO stage, LN status, and recurrence or not (all of them $P<0.001$ ). The higher stroma-rich proportions were found in EOC patients with advanced stage (36.13\% versus 19.75\%), LN metastasis (51.93\% versus $27.25 \%$ ), and recurrence (34.27\% versus 6.82\%). Stroma-rich EOC patients had obvious shorter median time of progressionfree survival (29 versus 39 months) and overall survival (50 versus 58 months), respectively. TSR was an independent prognostic factor for the evaluation of PFS in EOC. Stroma-rich tumors had worse prognosis and higher risk of relapse compared with those in stroma-poor tumors in EOC patients. Considered easy to determine for routine pathological examination, TSR may serve as a new prognostic histological parameter in EOC.
\end{abstract}

\section{Introduction}

Approximately, 95\% of ovarian cancers are of epithelial origin. In 2014, there were 21,980 estimated new diagnoses of ovarian cancer and 14,270 deaths from the disease, which is the most common cause of death among women with gynecologic cancer [1]. Tumor recurrence and metastasis are the leading cause of morbidity and mortality in EOC [2]. Traditionally, surgical pathological staging systems (International Federation of Gynecology and Obstetrics staging, FIGO staging) are still the most important tool for therapeutic decision-making in epithelial ovarian cancer (EOC). However, tumor cell pathological variables are only moderate indicators of outcome and therapy response. Since currently applied predictive factors do not adequately stratify risk in EOC patients, additional information is needed to individualize treatment [2].

Tumor invasion and metastasis are considered to be a multifactor process involving complex interactions of biological pathways [3]. The stroma surrounding cancer cells influences tumor development and behavior and the components of the tumor stroma have drawn increasing attention in predicting tumor prognosis [4]. Recently, as a consequence of the growing interest in the microenvironment, several studies have been conducted to evaluate the ratio of tumor to stroma (TSR) as a reflection of the microenvironment of cancer and survival outcome in esophageal cancer [5], breast cancer [6], colon cancer [7], and cervical cancer [8].

To our knowledge, the prognostic value of TSR has not been explored for EOC. Therefore, the objective of this study was to evaluate the prognostic value of TSR in EOC and its relationship with other prognostic factors.

\section{Materials and Methods}

2.1. Ethics Statement. Institutional review board approval was obtained for this study, and informed patient consent was waived owing to the retrospective nature of the study. 
2.2. Patient Population. In this retrospective study, 1065 patients diagnosed and treated for EOC between January 2001 and December 2011 at the Department of Gynecologic Oncology, Tianjin Medical University Cancer Institute and Hospital, were enrolled. Sixty-two patients were excluded with a previous history of cancer (ten patients with breast cancer, fifteen with colon cancer, twelve with rectum cancer, and twenty-five with other cancers). Due to the known effect of neoadjuvant therapy on stromal formation in tissue, we excluded 165 patients receiving neoadjuvant chemotherapy, which could interfere with the evaluation of TSR. Thus, 838 patients were enrolled for further analysis and, among them, 806 samples received adjuvant treatment after surgery.

Age, FIGO stage, histologic subtype, histologic grade, residual tumors, CA125, ascites volume, lymph node state, events of recurrences, and patient status at follow-up were extracted from available follow-up records.

2.3. Histopathological Scoring. Tissue samples consisting of $4 \mu \mathrm{m}$ haematoxylin-and-eosin- (H\&E-) stained sections from the most invasive part of the primary tumor were used for analysis using conventional microscopy. For TSR scoring, 2 investigators (Runfen Cheng and Yan Sun) estimated the TSR on all tumor slides and scored slides to the nearest 10 percentage points in a blinded manner. The most invasive tumor area of each slide was selected with the use of a $5 \mathrm{x}$ objective. The investigator chose a part of the sample containing both tumor and stromal tissue by using a $10 \mathrm{x}$ objective. Tumor cells had to be present at all borders of the image field. Mucinous tissue was visually excluded for scoring. For statistical analysis, the TSR was determined at the maximum discriminative power.

The tumor was evaluated per tenfold percentage (10, 20, $30 \%$, etc.), and TSR values of $10 \%$ and $100 \%$ were not seen. In case of an inconclusive score, a third observer was consulted. In case of tumor heterogeneity, areas with the lowest TSR value were considered decisive as is performed in routine pathology to determine tumor differentiation.

2.4. Follow-Up. Follow-up data were collected until death or December 2014. All patients had a regular follow-up schedule including a complete history, serum tumor marker detection, physical examination, and routine imaging evaluation every 3 months during the first 2 years since the last time of treatment and every 6 months thereafter. Overall survival (OS) was defined as the time interval from the date of primary surgery to the date of death (failure) or to the end of followup for women who were alive (censored). Progression-free survival (PFS) was defined as the time elapsed from the date of primary surgery to the appearance of disease recurrence or progression (failure) or the last follow-up for women who were alive with no evidence of disease recurrence or progression (censored).

2.5. Statistical Analysis. For statistical analysis, SPSS (Statistical Package for the Social Sciences) version 18.0 (Chicago, IL, USA) was applied. The results were considered statistically
TABLE 1: Clinicopathologic characteristics, demographics, and values for 838 EOC patients.

\begin{tabular}{|c|c|}
\hline Age (years) & Median: 55 ; range: $21-79$ \\
\hline $\operatorname{BMI}\left(\mathrm{kg} / \mathrm{m}^{2}\right)$ & Median: 23; range: $15-47$ \\
\hline \multicolumn{2}{|l|}{ Menopausal status } \\
\hline Yes & $505(60.3 \%)$ \\
\hline No & $333(39.7 \%)$ \\
\hline \multicolumn{2}{|l|}{ Histology } \\
\hline Serous & $599(71.5 \%)$ \\
\hline Mucous and others & $231(28.5 \%)$ \\
\hline \multicolumn{2}{|l|}{ Differentiation } \\
\hline $\mathrm{G}_{1-2}$ & $420(50.1 \%)$ \\
\hline $\mathrm{G}_{3}$ & $418(49.9 \%)$ \\
\hline \multicolumn{2}{|l|}{ FIGO stage (2009) } \\
\hline I-II & $243(29.0 \%)$ \\
\hline III-IV & $595(71.0 \%)$ \\
\hline With lymphadenectomy & $631(75.2 \%)$ \\
\hline Pelvic & $527(83.5 \%)$ \\
\hline Para-aortic + pelvic & $104(16.5 \%)$ \\
\hline Removed lymph nodes & Median: 25; range: $12-57$ \\
\hline \multicolumn{2}{|l|}{ Lymph nodes metastasis } \\
\hline No & $642(76.6 \%)$ \\
\hline Yes & $196(23.4 \%)$ \\
\hline \multicolumn{2}{|l|}{ Residual disease } \\
\hline$<1 \mathrm{~cm}$ & $631(75.3 \%)$ \\
\hline$\geq 1 \mathrm{~cm}$ & $207(24.7 \%)$ \\
\hline Ascites volume (mL) & Median: 1000 ; range: $0-7000$ \\
\hline$\leq 1000$ & $591(70.5 \%)$ \\
\hline$>1000$ & $247(29.5 \%)$ \\
\hline Serum CA125 (U/mL) & Median: 675 ; range: $23-7400$ \\
\hline$\leq 675$ & $521(62.2 \%)$ \\
\hline$>675$ & $317(37.8 \%)$ \\
\hline \multicolumn{2}{|l|}{ Tumor-stroma ratio (TSR) } \\
\hline Stroma-poor (TSR < 50\%) & $575(68.6 \%)$ \\
\hline Stroma-rich (TSR $\geq 50 \%$ ) & $263(31.4 \%)$ \\
\hline
\end{tabular}

significant with a probability of less than 0.05 . The chisquared and Fisher's exact tests were applied in analysis of categorical variable. The survival was determined by the Kaplan-Meier method, and the log rank test was used to determine significance. Factors that were deemed of potential importance by univariate analysis were included in the multivariate analysis by using Cox proportional hazard regression models. The prognostic significance of the TSR in EOC is also demonstrated by the subgroup analysis using the Cox proportional hazard regression model. Associations are shown as hazard ratios (HR) and $95 \%$ confidence intervals (CI).

\section{Results}

3.1. Patient Characteristics and Demographics. Clinicopathological characteristics of patients were shown in Table 1. In this study, a total of 838 patients with EOC were recruited 
TABLE 2: The determination of the best cutoff for tumor-stroma ratio (TSR) in EOC.

\begin{tabular}{|c|c|c|c|c|c|c|c|}
\hline TSR & Number & 3-year PFS & $\chi^{2}$ & $P$ & 5-year survival rate & $\chi^{2}$ & $P$ \\
\hline$<20 \%$ & 6 & 0 & \multirow{2}{*}{0.671} & \multirow{2}{*}{0.413} & 0 & \multirow{2}{*}{0.289} & \multirow{2}{*}{0.591} \\
\hline$\geq 20 \%$ & 832 & $48.2 \%$ & & & $40 \%$ & & \\
\hline$<30 \%$ & 91 & $52.5 \%$ & \multirow{2}{*}{3.240} & \multirow{2}{*}{0.072} & $47.2 \%$ & \multirow{2}{*}{4.312} & \multirow{2}{*}{0.038} \\
\hline$\geq 30 \%$ & 747 & $47.6 \%$ & & & $38.1 \%$ & & \\
\hline$<40 \%$ & 221 & $58.7 \%$ & \multirow{2}{*}{2.402} & \multirow{2}{*}{0.087} & $48.4 \%$ & \multirow{2}{*}{5.605} & \multirow{2}{*}{0.018} \\
\hline$\geq 40 \%$ & 617 & $54.2 \%$ & & & $37.1 \%$ & & \\
\hline$<50 \%$ & 575 & $55.3 \%$ & \multirow{2}{*}{13.704} & \multirow{2}{*}{$<0.001$} & $45.6 \%$ & \multirow{2}{*}{12.251} & \multirow{2}{*}{$<0.001$} \\
\hline$\geq 50 \%$ & 263 & $33.8 \%$ & & & $29.1 \%$ & & \\
\hline$<60 \%$ & 665 & $46.5 \%$ & \multirow{2}{*}{3.965} & \multirow{2}{*}{0.046} & $46.6 \%$ & \multirow{2}{*}{7.338} & \multirow{2}{*}{0.007} \\
\hline$\geq 60 \%$ & 173 & $42.2 \%$ & & & $34.4 \%$ & & \\
\hline$<70 \%$ & 717 & $47.8 \%$ & \multirow{2}{*}{3.058} & \multirow{2}{*}{0.080} & $40.2 \%$ & \multirow{2}{*}{3.778} & \multirow{2}{*}{0.052} \\
\hline$\geq 70 \%$ & 125 & $52.8 \%$ & & & $41.4 \%$ & & \\
\hline$<80 \%$ & 796 & $47.5 \%$ & \multirow{2}{*}{0.399} & \multirow{2}{*}{0.528} & $39.8 \%$ & \multirow{2}{*}{1.611} & \multirow{2}{*}{0.204} \\
\hline$\geq 80 \%$ & 42 & $69 \%$ & & & $53.2 \%$ & & \\
\hline$<90 \%$ & 824 & $48.4 \%$ & \multirow{2}{*}{0.103} & \multirow{2}{*}{0.749} & $40.3 \%$ & \multirow{2}{*}{0.992} & \multirow{2}{*}{0.319} \\
\hline$\geq 90 \%$ & 14 & $57.1 \%$ & & & $47.6 \%$ & & \\
\hline
\end{tabular}

PFS: progression-free survival.

Analysis was performed using the Kaplan-Meier method.

The minimum $P$ value was indicated in bold font.

between 2001 and 2011, and, among these patients, 207 cases have not received pelvic or para-aortic and pelvic lymphadenectomy owing to these patients with residual tumors $\geq 1 \mathrm{~cm}$. The median age of the 838 patients at the time of surgery was 55 years (range: 21-79 years) and the median follow-up time was 50 months (3-119 months).

3.2. The Optimal Cutoffs of TSR for EOC Patients. To determine the optimal cutoff of TSR, we analyzed the $P$ value for PFS and OS at different cutoffs in the statistical analysis and got the $50 \%$ level as the best cutoff point with maximum discriminating power for further analysis. Therefore, all patients were classified as "stroma-rich" or "stroma-poor" according to the proportion of stroma $\geq 50 \%$ or $<50 \%$, respectively (Table 2).

3.3. Relationships between TSR and Clinicopathological Variables in EOC Patients. As shown in Table 3, we divided the patients into two groups, stroma-rich group (TSR $\geq 50 \%$ ) and stroma-poor group (TSR $<50 \%$ ), and compared the difference of clinicopathological characteristics between the two groups (Figure 1). Chi-square test analysis revealed that there were no significant differences between the stroma-rich and stroma-poor groups regarding patient age, menopausal status, tumor histology, residual disease, serum CA125 level, and ascites volume. However, the proportions of stroma were significantly associated with FIGO stage, LN status, and recurrence or not. The higher stroma-rich proportions were found in EOC patients with advanced stage (36.13\% versus $19.75 \%$ ), LN metastasis (51.93\% versus $27.25 \%$ ), and recurrence (34.27\% versus $6.82 \%)$.
3.4. Survival and Multivariate Analysis. By the Kaplan-Meier method of univariate analysis, the shorter median of OS and PFS was related to advanced stage, LN metastasis, ascites volume $>1000 \mathrm{~mL}$, and stoma-rich type (all of them: $P<0.05$, Table 4). Furthermore, shorter median of PFS was also related to low differentiation $(P=0.001$, Table 4$)$ and shorter median of OS was related to serum CA125 $>675 \mathrm{U} / \mathrm{mL}(P=0.010$, Table 4).

These significant variables detected by univariate analysis were included in multivariate analysis. In Cox proportional hazard model, advanced stage and stroma-rich type were the independent factors for the evaluation of PFS $(P<0.05$, Table 4). Additionally, advanced stage and LN metastasis were the independent factors for the evaluation of OS $(P<$ 0.05 , Table 4 ).

Moreover, in the different subgroup analysis, according to the histology (serous and nonserous), FIGO stage (I-II and III-IV), LN status (metastasis and nonmetastasis), and residual disease $(<1$ and $\geq 1 \mathrm{~cm})$, TSR also was identified as the significant indicator for PFS and OS by using Cox univariate proportional hazard regression model (Table 5).

Generally, EOC patients with stroma-rich condition showed shorter PFS and OS than patients with stroma-poor condition. Kaplan-Meier survival curves are displayed in Figures 2(a) and 2(b).

\section{Discussion}

In the last decades, tumor cells have drawn the attention of the researchers as the main target for therapeutic interventions. However, evidence is growing that the peritumoral microenvironment plays key roles in tumor establishment 


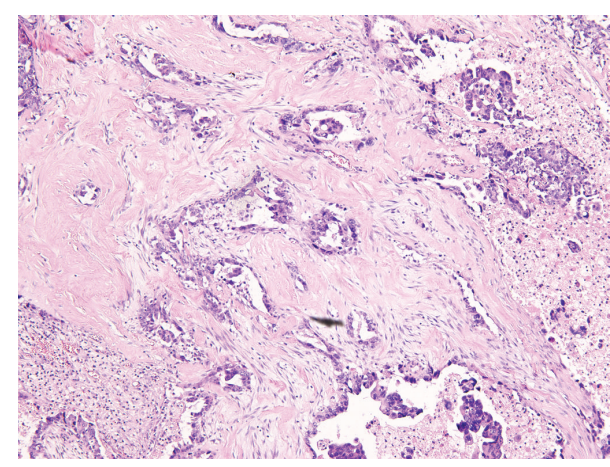

(a)

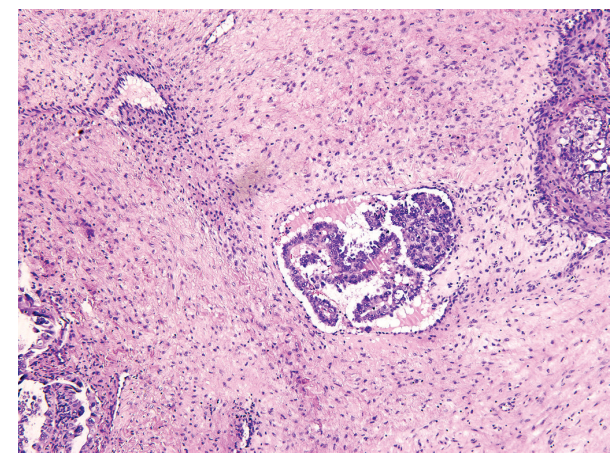

(c)

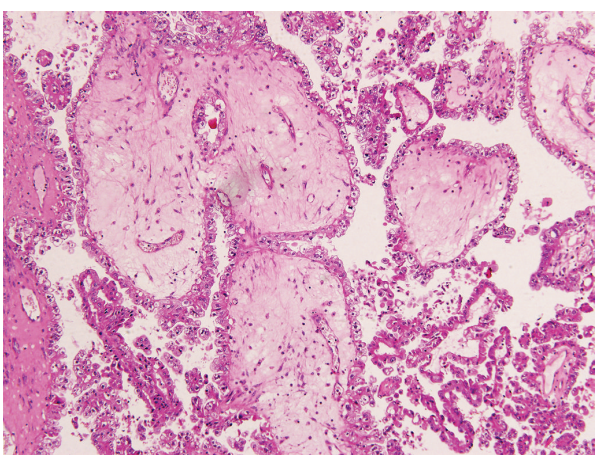

(e)

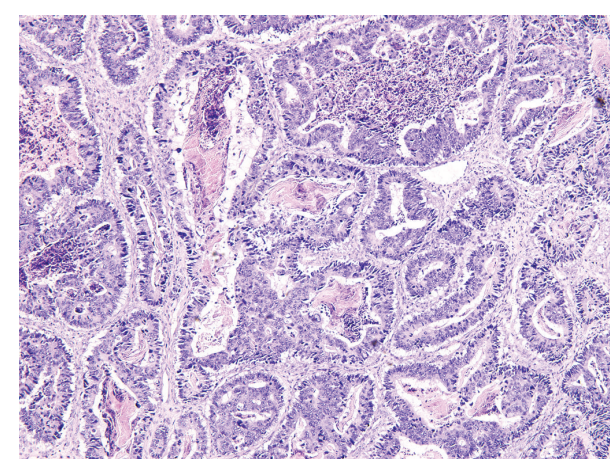

(b)

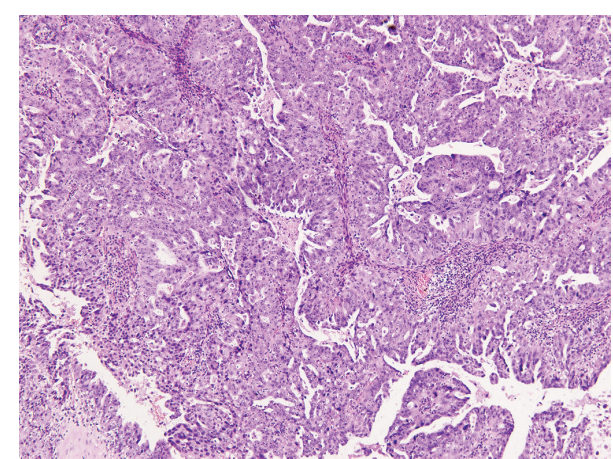

(d)

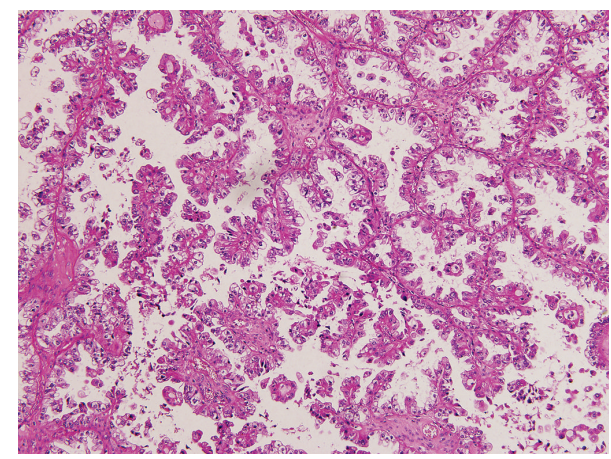

(f)

FIGURE 1: Representative images showing hematoxylin-and-eosin-stained $4 \mu \mathrm{m}$ sections of epithelial ovarian cancer (original magnification $\times 100)$. (a) Serous ovarian adenocarcinoma $\left(G_{2}\right)$ of stroma-rich type (TSR $\geq 50 \%$ ). (b) Serous ovarian adenocarcinoma $\left(G_{1}\right)$ staining of stromapoor type (TSR $<50 \%)$. (c) Serous ovarian adenocarcinoma $\left(G_{3}\right)$ of stroma-rich type (TSR $\left.\geq 50 \%\right)$. (d) Serous ovarian adenocarcinoma $\left(\mathrm{G}_{3}\right)$ of stroma-poor type (TSR < 50\%). (e) Clear cell ovarian adenocarcinoma of stroma-rich type (TSR $\geq 50 \%$ ). (f) Clear cell ovarian adenocarcinoma of stroma-poor type (TSR $<50 \%)$.

and tumor cell dissemination [9]. The mechanisms of the tumor-stroma interaction are critical in tumor progression, offering significant therapeutic implications [10]. TSR was first reported as an independent factor for survival in colon cancer [7]. In addition to being a newly identified prognostic factor, it was also confirmed to be significantly associated with prognosis of esophageal squamous cell carcinoma [5], breast cancer [6], and cervical cancer [8].

However, its prognostic role in EOC is largely unknown although TSR is a convenient and useful tool for pathologists to obtain more prognostic information from $\mathrm{H} \& \mathrm{E}$-stained slide. In this present study, we analyzed the prognostic value of TSR in 838 EOC patients. The optimal threshold level of TSR was determined on the basis of a maximum discriminating power for PFS and OS. We determined that $50 \%$ cutoff value was the most representative by use of statistical analysis. Therefore, all patients were classified as "stroma-rich" or "stroma-poor" according to the proportion of stroma $\geq 50 \%$ or $<50 \%$, respectively. The higher stromarich proportions were found in EOC patients with advanced stage, LN metastasis, and recurrence.

Tumor tissue is composed of both carcinoma cells and stromal cells recruited from normal tissue. Nowadays, accumulated lines of evidence had illustrated that, in normal 


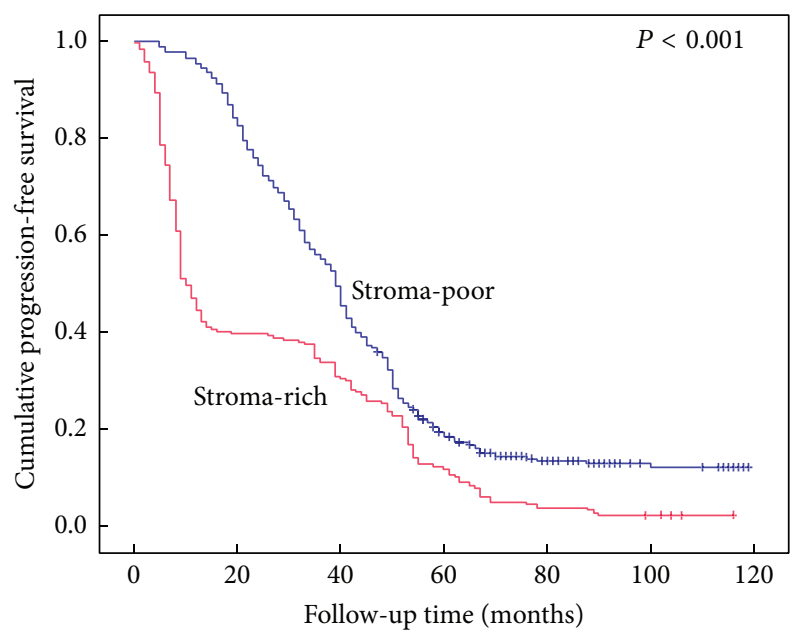

(a)

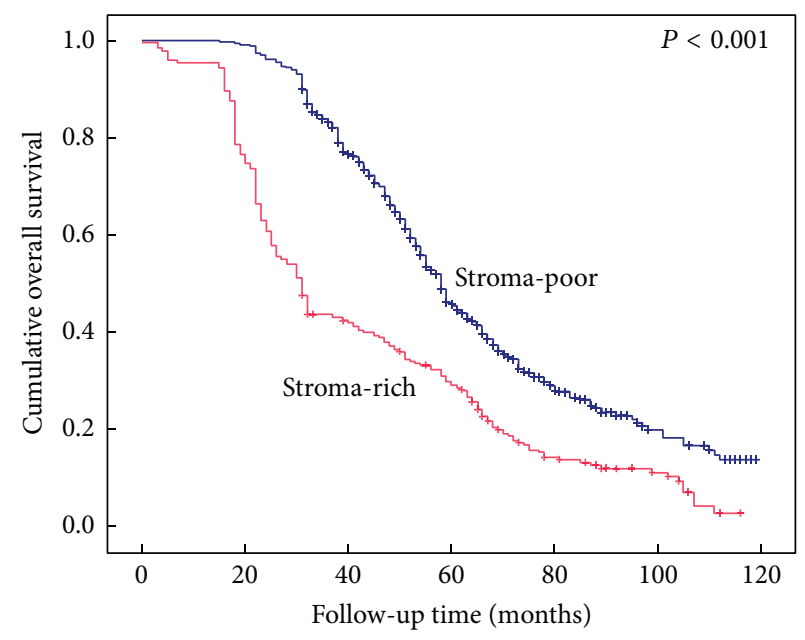

(b)

FIGURE 2: Kaplan-Meier curves for survival of 838 patients with epithelial ovarian cancer. Cumulative progression-free survival (a) and overall survival (b).

TABLE 3: Relationships between TSR and characteristics in 838 EOC patients.

\begin{tabular}{|c|c|c|c|c|c|}
\hline Characteristics & $\begin{array}{c}\text { Total } \\
(N=838) \\
\text { Number }(\%)\end{array}$ & $\begin{array}{l}\text { Stroma-poor } \\
\qquad(N=575) \\
\text { Number }(\%)\end{array}$ & $\begin{array}{l}\text { Stroma-rich } \\
(N=263) \\
\text { Number }(\%)\end{array}$ & $\begin{array}{l}\text { Stroma-rich proportion } \\
\qquad(N=263) \\
\text { Number }(\%)\end{array}$ & $P$ \\
\hline Age, year & & & & & 0.365 \\
\hline$\leq 55$ & $347(41.41)$ & $232(40.35)$ & $115(43.73)$ & $115(33.14)$ & \\
\hline$>55$ & $491(58.59)$ & $343(59.65)$ & $148(56.27)$ & $148(30.14)$ & \\
\hline Menopausal status & & & & & 0.820 \\
\hline Yes & $505(60.26)$ & $348(60.52)$ & $157(59.70)$ & $157(31.09)$ & \\
\hline No & $333(39.74)$ & $227(39.48)$ & $106(40.30)$ & $106(31.83)$ & \\
\hline Histology & & & & & 0.805 \\
\hline Serous & $599(71.48)$ & $409(71.13)$ & $190(72.24)$ & $190(31.72)$ & \\
\hline Mucous and others & $239(28.52)$ & $166(28.87)$ & $73(27.76)$ & $73(30.54)$ & \\
\hline Differentiation & & & & & 0.070 \\
\hline $\mathrm{G}_{1-2}$ & $420(50.12)$ & $276(48.00)$ & $144(54.75)$ & $144(34.29)$ & \\
\hline $\mathrm{G}_{3}$ & $418(49.88)$ & $299(52.00)$ & $119(50.42)$ & $119(28.47)$ & \\
\hline FIGO stage (2009) & & & & & $<0.001$ \\
\hline I-II & $243(29.00)$ & $195(33.91)$ & $48(18.25)$ & $48(19.75)$ & \\
\hline III-IV & $595(71.00)$ & $380(66.09)$ & $215(81.75)$ & $215(36.13)$ & \\
\hline Lymph nodes metastasis & & & & & $<0.001$ \\
\hline No & $657(78.40)$ & $478(83.13)$ & $179(68.06)$ & $179(27.25)$ & \\
\hline Yes & $181(21.60)$ & $87(16.87)$ & $94(31.94)$ & $94(51.93)$ & \\
\hline Residual disease & & & & & 0.796 \\
\hline$<1 \mathrm{~cm}$ & $631(75.30)$ & $431(74.96)$ & $200(76.05)$ & $200(31.70)$ & \\
\hline$\geq 1 \mathrm{~cm}$ & $207(24.70)$ & $144(25.04)$ & $63(23.95)$ & $63(30.43)$ & \\
\hline Ascites volume $(\mathrm{mL})$ & & & & & 0.464 \\
\hline$\leq 1000$ & $591(70.53)$ & $410(71.30)$ & $181(68.82)$ & $181(30.63)$ & \\
\hline$>1000$ & $247(29.47)$ & $165(28.70)$ & $82(31.18)$ & $82(33.20)$ & \\
\hline Serum CA125 (U/mL) & & & & & 0.091 \\
\hline$\leq 675$ & $521(62.17)$ & $346(60.17)$ & $175(66.54)$ & $175(33.59)$ & \\
\hline$>675$ & $317(37.83)$ & $229(39.83)$ & $88(33.46)$ & $88(27.76)$ & \\
\hline Recurrence & & & & & $<0.001$ \\
\hline No & $88(10.50)$ & $82(14.26)$ & $6(2.28)$ & $6(6.82)$ & \\
\hline Yes & $750(89.50)$ & $493(85.74)$ & $257(97.72)$ & $257(34.27)$ & \\
\hline
\end{tabular}




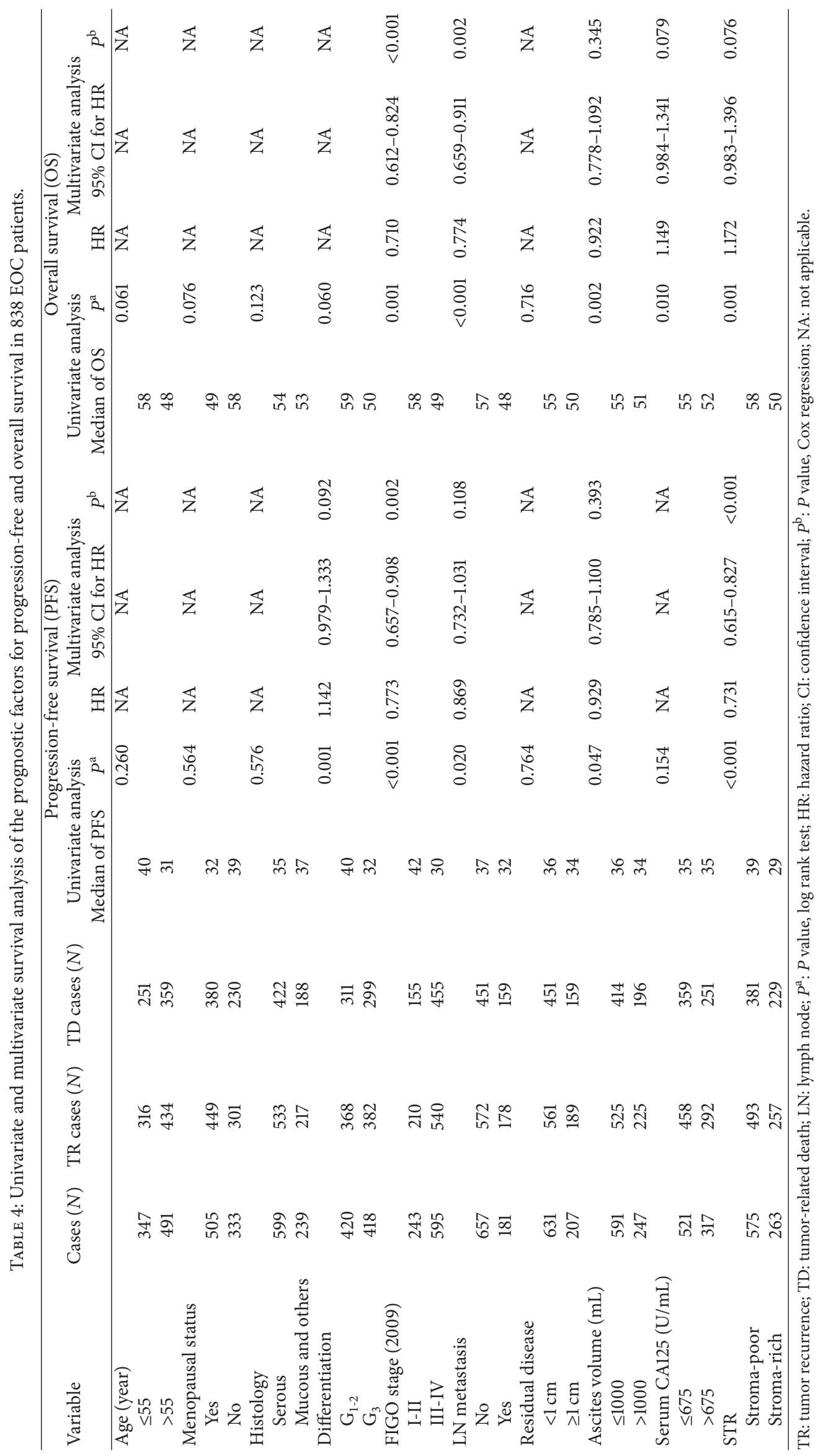


TABLE 5: The significance of TSR in 838 EOC patients according to different subgroup using multivariate Cox survival analysis.

\begin{tabular}{|c|c|c|c|c|c|c|c|c|c|}
\hline \multirow{3}{*}{ Variable } & \multirow{3}{*}{ Cases $(N)$} & \multirow{3}{*}{$\operatorname{TR}(N)$} & \multirow{3}{*}{$\mathrm{TD}(N)$} & \multicolumn{6}{|c|}{ TSR } \\
\hline & & & & \multicolumn{3}{|c|}{ Progression-free survival (PFS) } & \multicolumn{3}{|c|}{ Overall survival (OS) } \\
\hline & & & & HR & $95 \% \mathrm{CI}$ & $P$ & HR & $95 \% \mathrm{CI}$ & $P$ \\
\hline \multicolumn{10}{|l|}{ Histology } \\
\hline Serous & 599 & 533 & 422 & 1.873 & $1.564-2.242$ & $<0.001$ & 2.075 & $1.702-2.530$ & $<0.001$ \\
\hline Mucous and others & 239 & 217 & 188 & 1.616 & $1.210-2.157$ & 0.001 & 1.860 & $1.348-2.567$ & $<0.001$ \\
\hline \multicolumn{10}{|l|}{ FIGO stage (2009) } \\
\hline I-II & 243 & 210 & 155 & 1.065 & $0.765-1.481$ & 0.710 & 1.038 & $0.705-1.528$ & 0.849 \\
\hline III-IV & 595 & 540 & 455 & 2.058 & $1.726-2.455$ & $<0.001$ & 2.302 & $1.904-2.783$ & $<0.001$ \\
\hline \multicolumn{10}{|l|}{ LN metastasis } \\
\hline No & 657 & 572 & 451 & 1.276 & $1.082-1.505$ & 0.004 & 1.818 & $1.492-2.216$ & $<0.001$ \\
\hline Yes & 181 & 178 & 159 & 2.156 & $1.588-2.926$ & $<0.001$ & 2.555 & $1904-2.783$ & $<0.001$ \\
\hline \multicolumn{10}{|l|}{ Residual disease } \\
\hline$<1 \mathrm{~cm}$ & 631 & 561 & 451 & 1.961 & $1.646-2.337$ & $<0.001$ & 2.167 & $1.790-2.624$ & $<0.001$ \\
\hline$\geq 1 \mathrm{~cm}$ & 207 & 189 & 159 & 1.387 & $1.015-1.896$ & 0.040 & 1.607 & $1.135-2.276$ & 0.007 \\
\hline
\end{tabular}

TSR: tumor-stroma ratio; TR: tumor recurrence; TD: tumor-related death; LN: lymph node; HR: hazard ratio; CI: confidence interval; $P: P$ value, Cox regression.

tissue, the stroma may actually act as a barrier in tumorigenesis by constraining tumor cell proliferation [11, 12]. In tumor tissue, however, stromal components, the main part of tumor microenvironment, could facilitate the process of tumor progression [13]. The mechanism underlying tumorpromoting effect of stroma is still not fully understood. Previous evidence supports the notion that the increase in abundance of fibroblasts in tumor causes deposition of fibrotic extracellular matrix (ECM). Changes in ECM structure can be further stimulated by proteases, which degrade stroma. Together, this results in disruption of epithelial tissue and remodeling of the ECM, facilitating invasion of tumors cells [14].

Recently, the so-called cancer-associated fibroblasts (CAFs) composed of the major cellular components of tumor stroma caused more and more attention, which were found to have a predominant role in tumor growth and progression [15]. The CAF-derived regulators and extracellular matrix proteins can support cancer progression by providing a protective microenvironment for the cancer cells via reduction of chemotherapy sensitivity [16]. CAFs are frequently observed in the stroma of human carcinoma and secrete a variety of soluble factors such as transforming growth factor beta 1 (TGF- $\beta 1$ ) [17], stromal cell-derived factor 1, and other soluble factors, which act in a paracrine manner and affect not only cancer cells but also other cell types present in the stroma [18]. As a sign of their activation, CAFs produce several mesenchyme-specific proteins such as fibroblast-specific protein (FSP-1), fibroblast-activating protein (FAP), vimentin, and alpha-smooth muscle actin $(\alpha$-SMA), the prototypical marker for myofibroblasts. CAFs are also a rich source of different secreted factors such as cytokines and chemokines (e.g., IL-6, CXCL8, and CXCL12) and growth factors like epidermal growth factor (EGF) and vascular endothelial-derived growth factor (VEGF), which could promote angiogenesis, which is essential for tumor growth and progression $[19,20]$. Additionally, CAFs have recently been investigated for their function as a regulator of immune cell recruitment and function [21]. Previous studies suggest CAFs are first educated by immune cells during the initial stages of tumorigenesis but they acquire the ability to recruit and regulate immune cells to an eventually immune-suppressed phenotype that is compatible with disease progression [22]. Moreover, a recent study by Herrera and coworkers investigating the role of CAFs in colon cancer suggested that the combination of CAFs and M2 macrophage signatures correlated with a clear difference in disease progression and survival of advanced stage patients [23].

Significantly, our results manifested the fact that EOC patients with stroma-rich condition had obvious shorter PFS and OS. Importantly, we revealed that TSR may be an independent and strong prognostic factor for the evaluation of PFS in EOC patients. However, our study has its shortcomings, which was retrospective study. Moreover, the mechanism underlying tumor-promoting effect of tumor in EOC was still not explored by us. It is of greater value to conduct a prospective study and investigate the molecular mechanism, which may avail us to determine whether the TSR could be used in clinical practice for better risk classification of EOC patients and even for implementation in standard pathology reports in the future.

Conclusively, our findings indicate EOC patients could be classified as "stroma-rich" or "stroma-poor" according to the best cutoff of TSR 50\%. The higher stroma-rich proportions were found in EOC patients with advanced stage, LN metastasis, and recurrence. TSR may be an independent and strong prognostic factor for the evaluation of PFS in EOC patients. The TSR is easy to determine, reproducible, and quickly performed by using routine pathological examination on H\&E-stained sections. Thus, the study of tumor stroma has potential to facilitate the prognostic assessment of EOC 
patients in the clinical practice and even in combination with other therapeutic agents for individual treatment in the future.

\section{Conflict of Interests}

None of the authors has any potential conflict of interests.

\section{Acknowledgments}

The authors thank the two pathologists Runfen Cheng and Yan Sun for the careful evaluations of TSR in this study. This work was supported by Tianjin Health Bureau of Science and Technology Funds (2012KZ073) and the National Natural Science Foundation (81302250).

\section{References}

[1] R. Siegel, J. Ma, Z. Zou, and A. Jemal, "Cancer statistics, 2014," CA-Cancer Journal for Clinicians, vol. 64, no. 1, pp. 9-29, 2014.

[2] G. C. Jayson, E. C. Kohn, H. C. Kitchener, and J. A. Ledermann, "Ovarian cancer," The Lancet, vol. 384, no. 9951, pp. 1376-1388, 2014.

[3] F. Klemm and J. A. Joyce, "Microenvironmental regulation of therapeutic response in cancer," Trends in Cell Biology, vol. 25, no. 4, pp. 198-213, 2015.

[4] D. Tarin, "Role of the host stroma in cancer and its therapeutic significance," Cancer and Metastasis Reviews, vol. 32, no. 3-4, pp. 553-566, 2013.

[5] K. Wang, W. Ma, J. Wang et al., "Tumor-stroma ratio is an independent predictor for survival in esophageal squamous cell carcinoma," Journal of Thoracic Oncology, vol. 7, no. 9, pp. 14571461, 2012.

[6] E. M. de Kruijf, J. G. H. van Nes, C. J. H. van de Velde et al., "Tumor-stroma ratio in the primary tumor is a prognostic factor in early breast cancer patients, especially in triple-negative carcinoma patients," Breast Cancer Research and Treatment, vol. 125, no. 3, pp. 687-696, 2011.

[7] A. Huijbers, R. A. E. M. Tollenaar, G. W. V. Pelt et al., "The proportion of tumor-stroma as a strong prognosticator for stage II and III colon cancer patients: validation in the victor trial," Annals of Oncology, vol. 24, no. 1, pp. 179-185, 2013.

[8] J. Liu, J. Liu, J. Li et al., "Tumor-stroma ratio is an independent predictor for survival in early cervical carcinoma," Gynecologic Oncology, vol. 132, no. 1, pp. 81-86, 2014.

[9] A. Casazza, G. Di Conza, M. Wenes, V. Finisguerra, S. Deschoemaeker, and M. Mazzone, "Tumor stroma: a complexity dictated by the hypoxic tumor microenvironment," Oncogene, vol. 33, no. 14, pp. 1743-1754, 2014.

[10] O. De Wever and M. Mareel, "Role of tissue stroma in cancer cell invasion," The Journal of Pathology, vol. 200, no. 4, pp. 429-447, 2003.

[11] N. Bezdenezhnykh, N. Semesiuk, O. Lykhova, V. Zhylchuk, and Y. Kudryavets, "Impact of stromal cell components of tumor microenvironment on epithelial-mesenchymal transition in breast cancer cells," Experimental Oncology, vol. 36, no. 2, pp. 72-78, 2014.

[12] Y. Mao, E. T. Keller, D. H. Garfield, K. Shen, and J. Wang, "Stromal cells in tumor microenvironment and breast cancer," Cancer and Metastasis Reviews, vol. 32, no. 1-2, pp. 303-315, 2013.
[13] M. J. Bissell and D. Radisky, "Putting tumours in context," Nature Reviews Cancer, vol. 1, no. 1, pp. 46-54, 2001.

[14] G. S. Wong and A. K. Rustgi, "Matricellular proteins: priming the tumour microenvironment for cancer development and metastasis," British Journal of Cancer, vol. 108, no. 4, pp. 755761, 2013

[15] M. Augsten, "Cancer-associated fibroblasts as another polarized cell type of the tumor microenvironment," Frontiers in Oncology, vol. 4, article 62, 2014.

[16] G. Valcz, F. Sipos, Z. Tulassay, B. Molnar, and Y. Yagi, "Importance of carcinoma-associated fibroblast-derived proteins in clinical oncology," Journal of Clinical Pathology, vol. 67, no. 12, pp. 1026-1031, 2014.

[17] A. Calon, D. V. F. Tauriello, and E. Batlle, "TGF-beta in CAFmediated tumor growth and metastasis," Seminars in Cancer Biology, vol. 25, pp. 15-22, 2014.

[18] J. Massagué, “TGF $\beta$ in cancer," Cell, vol. 134, no. 2, pp. 215-230, 2008.

[19] E. L. Spaeth, J. L. Dembinski, A. K. Sasser et al., "Mesenchymal stem cell transition to tumor-associated fibroblasts contributes to fibrovascular network expansion and tumor progression," PLoS ONE, vol. 4, no. 4, Article ID e4992, 2009.

[20] H. Sugimoto, T. M. Mundel, M. W. Kieran, and R. Kalluri, "Identification of fibroblast heterogeneity in the tumor microenvironment," Cancer Biology \& Therapy, vol. 5, no. 12, pp. 16401646, 2006.

[21] J. Harper and R. C. A. Sainson, "Regulation of the anti-tumour immune response by cancer-associated fibroblasts," Seminars in Cancer Biology, vol. 25, pp. 69-77, 2014.

[22] T. Udagawa and M. Wood, "Tumor-stromal cell interactions and opportunities for therapeutic intervention," Current Opinion in Pharmacology, vol. 10, no. 4, pp. 369-374, 2010.

[23] M. Herrera, A. Herrera, G. Domínguez et al., "Cancerassociated fibroblast and M2 macrophage markers together predict outcome in colorectal cancer patients," Cancer Science, vol. 104, no. 4, pp. 437-444, 2013. 


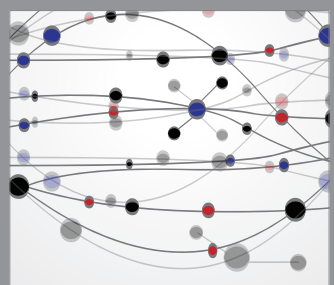

The Scientific World Journal
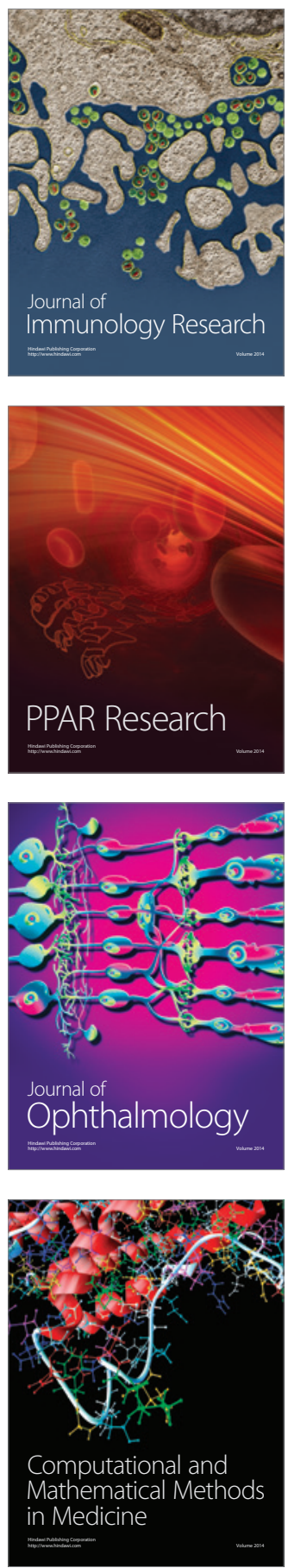

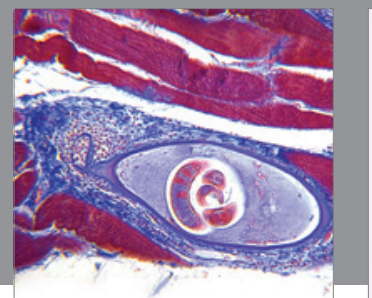

Gastroenterology

Research and Practice
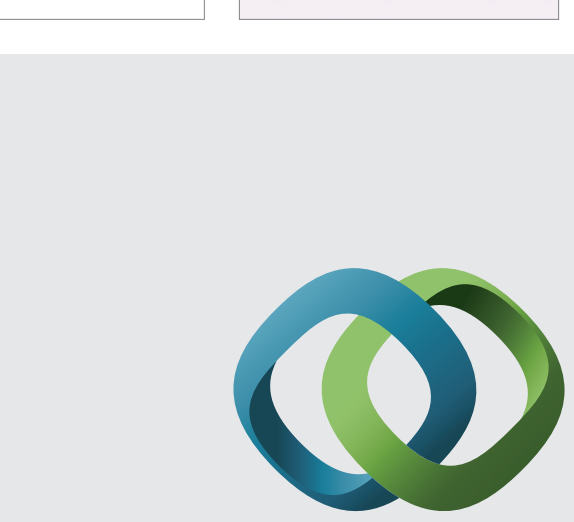

\section{Hindawi}

Submit your manuscripts at

http://www.hindawi.com
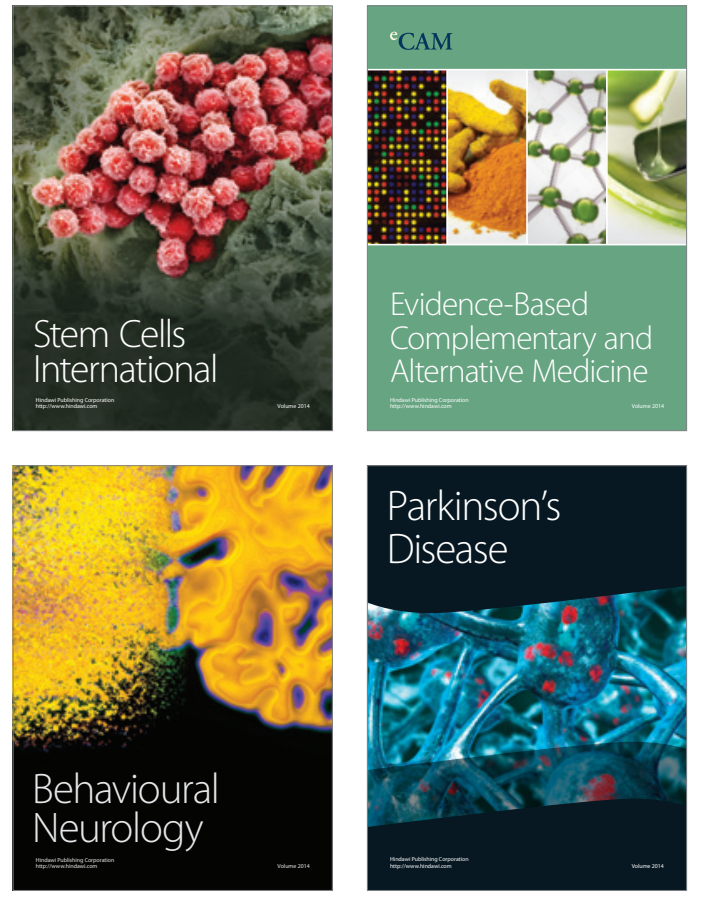
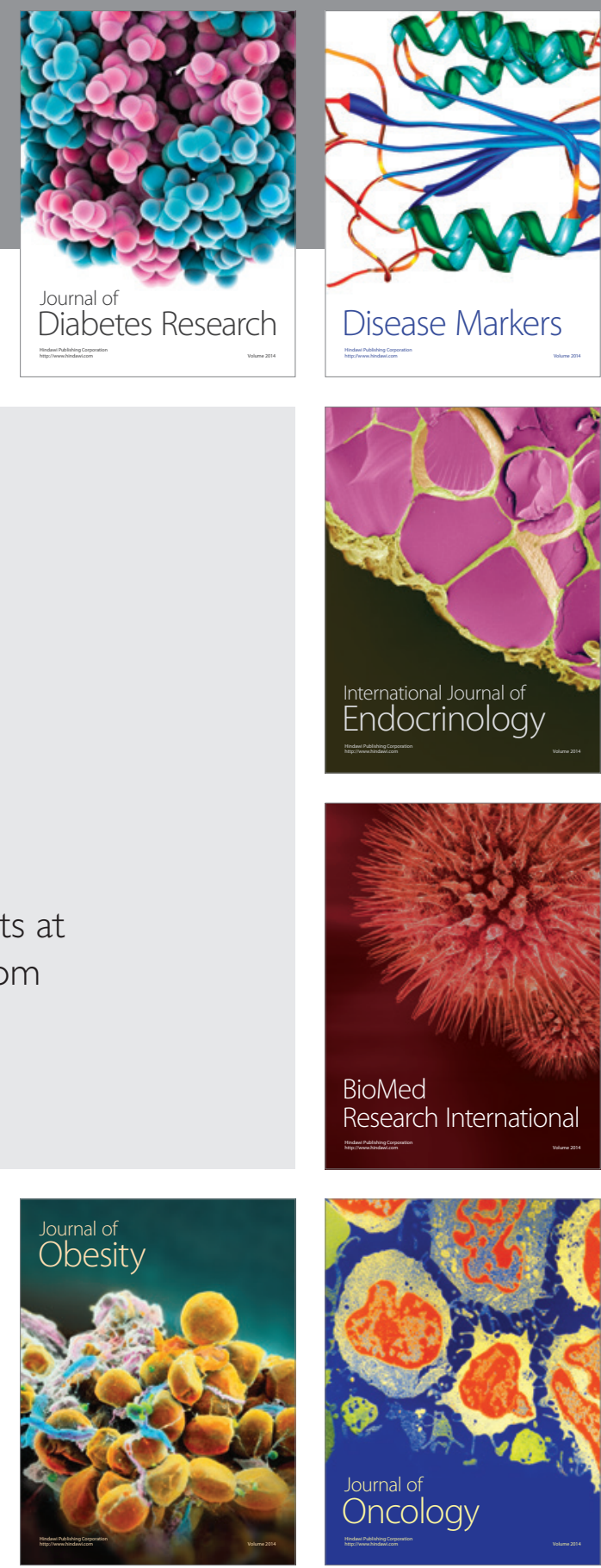

Disease Markers
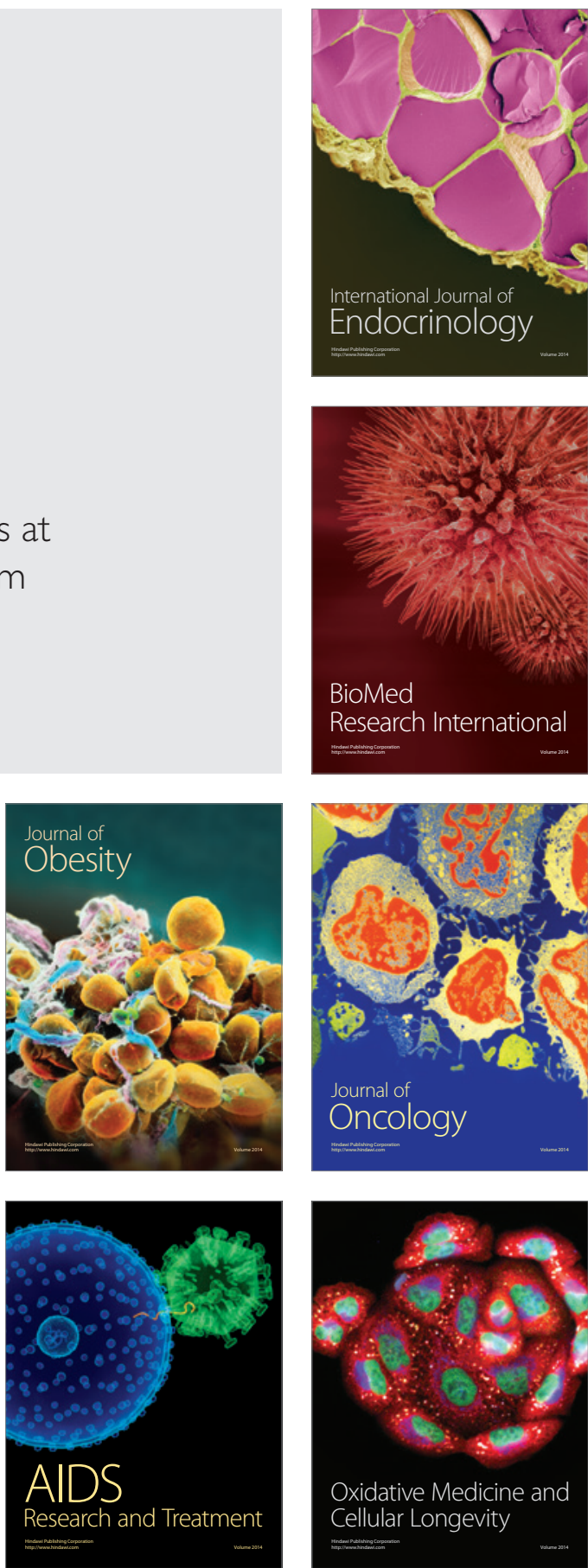